\title{
TRES NUEVOS COBRES DE VICTOR WOLFVOET CON LA PAZ Y LA GUERRA BAJO LAS CONSIGNA DE RUBENS
}

\author{
MATÍAS DíAZ PADRÓN
}

\begin{abstract}
Tres cobres más se añaden a la escasa producción conocida hasta ahora del último de los discípulos de Rubens. Identificado por el estilo, al que se une el contenido y la intención propagandística contra la guerra que asola Europa. Rubens busca la concordia y la paz. Su discípulo Victor Wolfvoet contribuye a la divulgación de estos ideales.

Palabras clave: Escuela de Amberes; Alegoría del buen gobierno; Minerva; Ceres; Hércules; Marte.
\end{abstract}

\section{THREE NEW PAINTINGS ON COPPER BY VICTOR WOLFVOET WITH PEACE AND WAR UNDER THE GUIDANCE OF RUBENS}

Three more works on copper are added to the until-now little known production of the last Rubens disciple. They have been identified on the basis of style united with their content and propagandistic intent against the war devastating Europe. Rubens seeks reconciliation and peace, and his disciple Victor Wolfvoet contributes to the spread of these ideals.

Key words: Antwerp School; Allegory of Good Government; Minerva; Ceres; Hercules; Mars.

Tratamos en estas mismas páginas de tres cobres restituidos a Victor Wolfvoet por motivos de técnica y estilo en distintas instituciones y colecciones españolas ${ }^{1}$. Apuntamos algunas precisiones de su personalidad que razonablemente puede incluirle en la pintura de género de la escuela de Amberes en el siglo XVII, tanto por el formato, el soporte en cobre, como por la intención decorativa marginal al contenido ${ }^{2}$. Es un copista irredento de Rubens con afines modelos y afines historias devotas y mitológicas, con igual gusto por el movimiento de las formas en consonancia siempre con las composiciones del maestro.

Igual que los cobres publicados, las características en estos reunidos aquí, son las mismas: fidelidad reverencial al maestro con sutil cambio en los modelos, los rasgos, el modelado y las sombras. No insistimos más en esto, pero sí en el número de obras localizadas en la Península, que a la altura de nuestras indagaciones parece evidente un contacto muy directo con los agentes de Flandes por la vía comercial del Norte y Sur de la Península Ibérica. Sin embargo, ni las pinturas en concreto, ni su nombre, aparecen en los inventarios y registros de la corona y la nobleza. La proliferación de sus pinturas es notable sin embargo en parroquias, y residencias de mediano estatus. No obstante dos de los cobres que estudiamos alusivos a la Paz y la Guerra nos llegan de extramuros: uno en colección privada de Barcelona, con atribución a Cornelis Schut y otro a Guilliam Forchondt el Joven. El tercero con los desastres de la guerra procede del coleccionismo histórico español, por su origen y procedencia en tierras sevillanas, posiblemente importado de los Países Bajos en la ruta del Sur con los centros más activos de Sevilla, Málaga y Cádiz. Esta tercera pintura estuvo expuesta entre los anónimos discípulos de Rubens en la exposición homenaje del IV Centenario del nacimiento de Rubens, en Sevilla.

Todos son asuntos angustiosos y asfixiantes en el nudo gordiano de las guerras de España por la hegemonía de Europa. Todos responden a la temática de Rubens con la queja a los males de la guerra que asoló aquella Europa en conflicto. España está implicada en lo más profundo de aquella contienda. Esto es sobradamente conocido ${ }^{3}$. Los tres cobres propagan bocetos y pinturas diseñados por Rubens. Esto lo hemos dicho, pero también los ideales de paz que Rubens infunde

\footnotetext{
1 DíAZ PADRÓn, 2006: 403.

2 DíAZ PADRÓN, 1999: 323.

3 BADOUIN, 1977: 233.
} 
con igual pasión en sus obras con el lenguaje directo de sus pinceles. No hay duda de la función trascendente de este discípulo menor implicado en el programa que Rubens comunicó a su amigo Joost Susterman con la sinceridad que anida en su alma. Los tres cobres propagan la paz que soñaba.

No hay duda de la intención de los cobres de este digno discípulo de Rubens que divulgaba el programa político que Rubens confesó a su amigo Pierre Dupuy en carta del 28 de mayo de 1627 desde París: "nuestra villa languidece como un cuerpo que se consume poco a poco. Todos los días vemos caer su población. Nuestro pueblo no dispone de ningún medio para sobrevivir a su sustento" 4 . Los tres cobres transmiten con igual fortuna el mensaje con la forma y la expresión. La ejecución es audaz y fácil pero lejos del ardor cromático, la pasión y brillo de los originales de Rubens.

En fin, es la súplica por la paz que Rubens intentó con sus escasos medios en las misiones que le encarga la Archiduquesa desde Flandes en Madrid y Londres (1628-1630). Veía su pueblo consumido en la guerra por la política belicista del Conde Duque de Olivares frente a los anhelos de paz de Isabel Clara Eugenia y Ambrosio Spínola. Es la atmósfera que traspiran estos cobres de Victor Wolfvoet. Intención y mensaje en fecha afín a los originales de Rubens. Esto nos permite fechar los cobres en el mismo tiempo que los acontecimientos que se ventilan. Podría hacerse con holgura entre 1637 y la muerte de Victor Wolfvoet (1612-1652).

El primero de los cobres con la Alegoría de la Guerra y la Paz (o Alegoría del buen gobierno) (fig. 1), se cataloga en el taller de Cornelis Schut por la casa Sotheby's ${ }^{5}$. Pintor que cuenta hoy con monografía ${ }^{6}$. Aunque vinculado con los satélites de Rubens, Cornelis Schut no se somete en la medida de otros condiscípulos al estilo del maestro. Tampoco es normal la utilización del cobre como soporte en su producción, por el contrario habitual en Victor Wolfvoet, salvo en las guirnaldas de Daniel Seghers. Quizá pudo confundir la distribución de los personajes en diagonales osadas, típico en Cornelis Schut. Nos parece obligado al llegar a esta anotación técnica traer a colación una Anunciación que le atribuye la profesora Hairs, a quien debemos las investigaciones más importantes de la escuela de Amberes ${ }^{7}$. Pienso que también se trata de un original de Victor Wolfvoet que espero tratar en otro momento.

En la Alegoría de la Guerra y la Paz protagoniza la acción Minerva, diosa de la sabiduría, de las ciencias y las artes. Es la Atenea romana ocupando el eje de la composición, sentada sobre el mundo que protege de las miserias de la guerra. De hecho, garantiza la paz como postula el grabado de $\mathrm{Ph}$. Galle según Martin de $\operatorname{Vos}^{8}$. Utiliza los mismos símbolos derramados entre cadáveres en tierra. Marte aplasta con sus pies un cadáver en escorzo de orejas puntiagudas: es la ignorancia vencida. Una figura alegórica que Rubens utiliza en los Triunfos de la Eucaristía. Equilibra la composición con la Fortuna, entre hortalizas y frutos de la tierra a la derecha, volviendo la mirada a la diosa que le protege. La Agricultura está representada junto a una pala semioculta, símbolo de su función. Son los gestos y semblantes lo que habla con más elocuencia de la diosa protectora de la Agricultura con sus brazos entrelazados y mirada desafiando a Marte. La Victoria figura ascendiendo en diagonal con la corona de laurel.

Todos los colores de los mantos y túnicas son diferentes: azules, amarillos, verdes y rojos en contraste con el duro metal de la armadura de Marte, y sin embargo logra la armonía tonal en ósmosis con el espacio. Es algo que Victor Wolfvoet aprende de Rubens. Es un acierto la luz frontal desde lo alto y la valoración del espacio con la diosa de la paz y la fertilidad.

\footnotetext{
4 Rooses y Ruelens, 1887-1909: 252.

5 Sotheby's Amsterdam, 14-XI-1990 n. ${ }^{\circ} 39$.

6 WiLMERS, 1996.

7 HAIRS, 1968: 221-222.

8 Hollstein's, 1995: 154.
} 


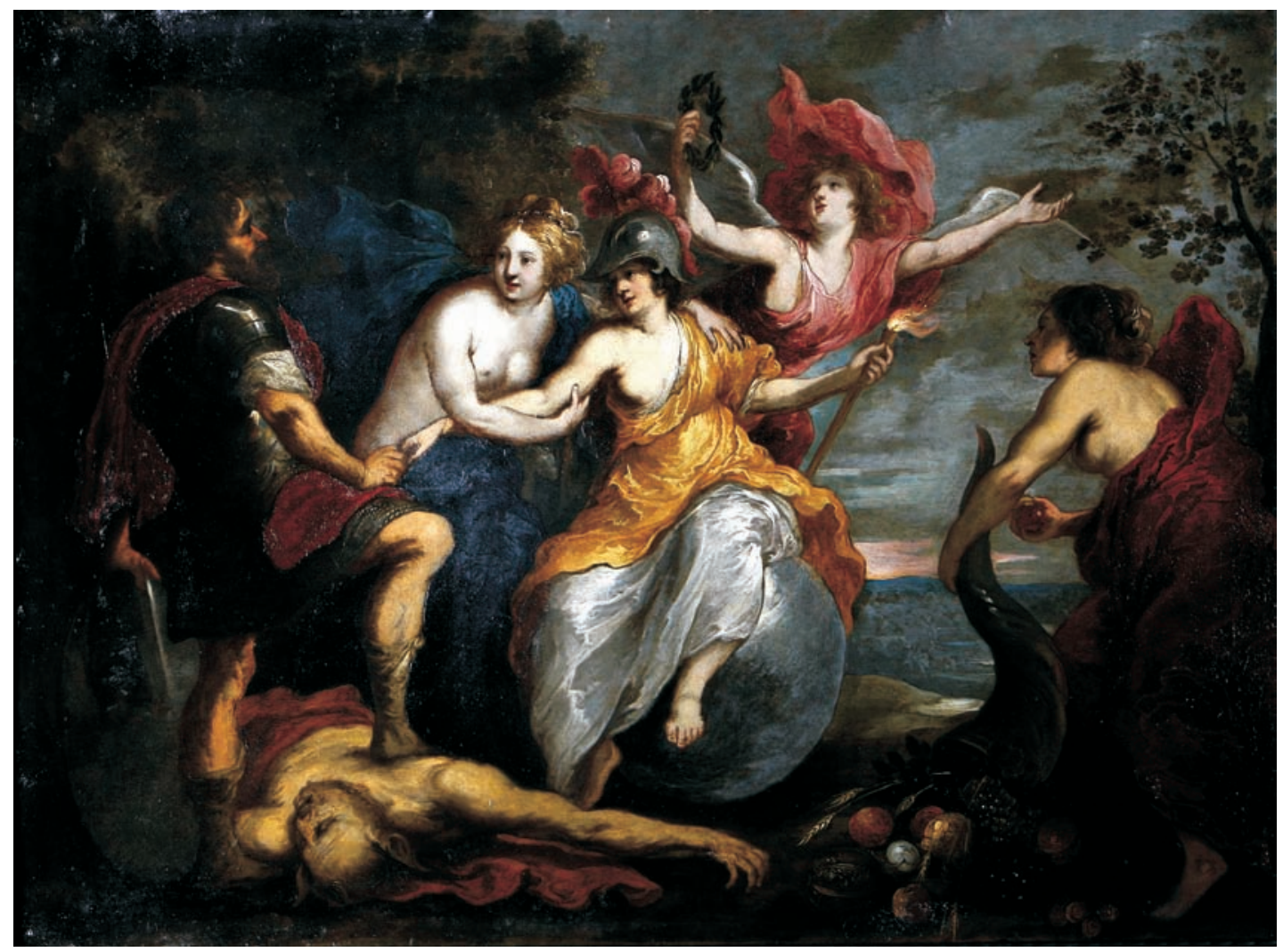

Fig. 1. Victor Wolfvoet, Alegoría de la Guerra y la Paz, Barcelona.

De la composición no ha llegado a nosotros el lienzo original de Rubens, que quizá no llevó a buen término. Quizá sólo el proyecto o "modeletto" como apuntó Held, tratando de la Alegoría de Minerva enfrentada con Marte correspondiente al tercero de los cobres que tratamos en este artículo.

La segunda réplica de la Alegoría de la Guerra y la Paz (fig. 2) fue adquirida en la galería Sothebys de Londres con atribución a Guilliam Forchondt ${ }^{9}$. Es un pintor de escasa producción y exiguos estudios ${ }^{10}$. Pero dista del estilo y la técnica de este cobre que restituimos a Victor Wolfoet. Esta segunda alegoría nos brinda las mismas características de la primera, por lo que no es preciso repetirlas. Basta el cotejo de una con otra. Las diferencias son insignificantes: el perfil más huidizo del rostro de Marte y menos fulgurante la antorcha encendida que porta Minerva y más amplio el boscaje en el ángulo alto izquierdo y espaldas de la Fortuna. También tiene mayor luminosidad el color de la superficie a diferencia de la primera réplica, poco generosa en el espesor de la preparación.

El tercer cobre con Hércules y Minerva enfrentados a Marte (fig. 3) defendiendo la madre a los niños arrasados por el dios de la Guerra fue expuesto en Sevilla ${ }^{11}$. Una réplica idéntica posee

\footnotetext{
9 SotheBY's, 10-VII-2008, n. ${ }^{\circ} 141$.

10 Duverger, 1989-1991: 303; Mesa y Gisbert, 1992: 182, 377; Díaz Padrón, Ms. 1976: 1339.

11 Homenaje..., 1978.
} 


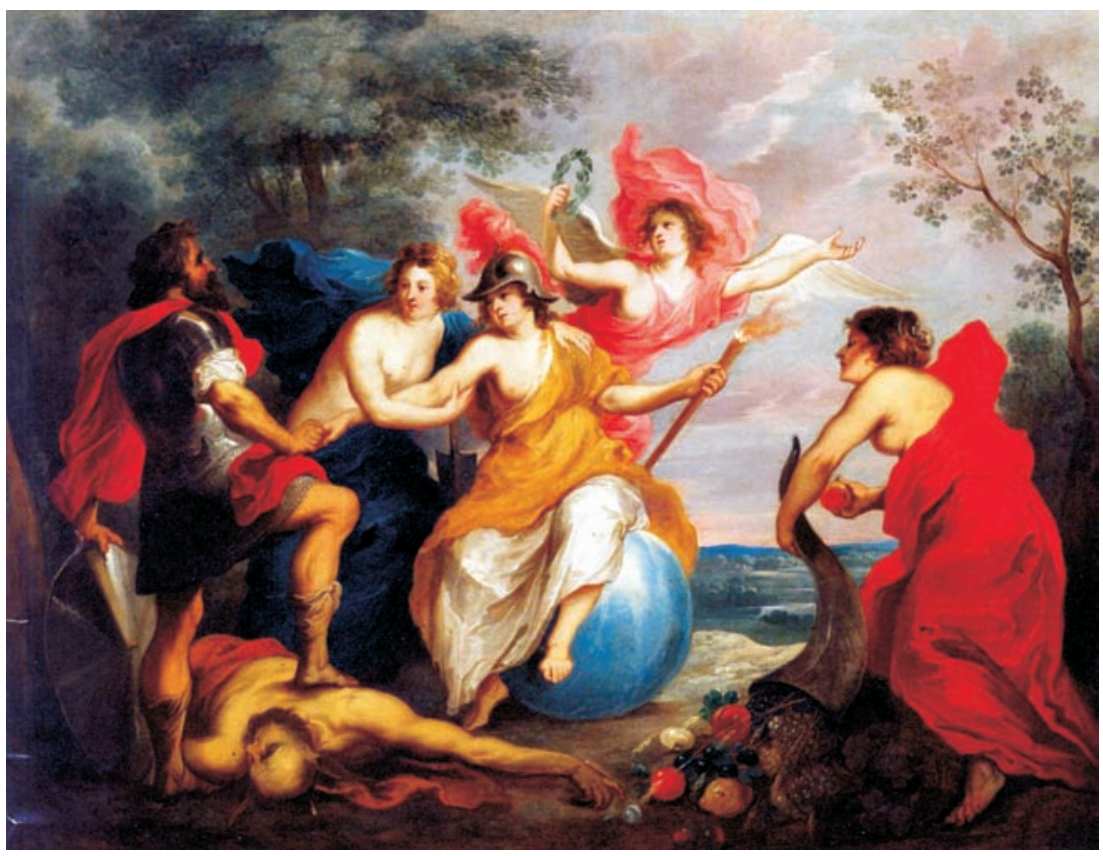

Fig. 2. Victor Wolfvoet, Alegoría de la Guerra y la Paz, Berlín.

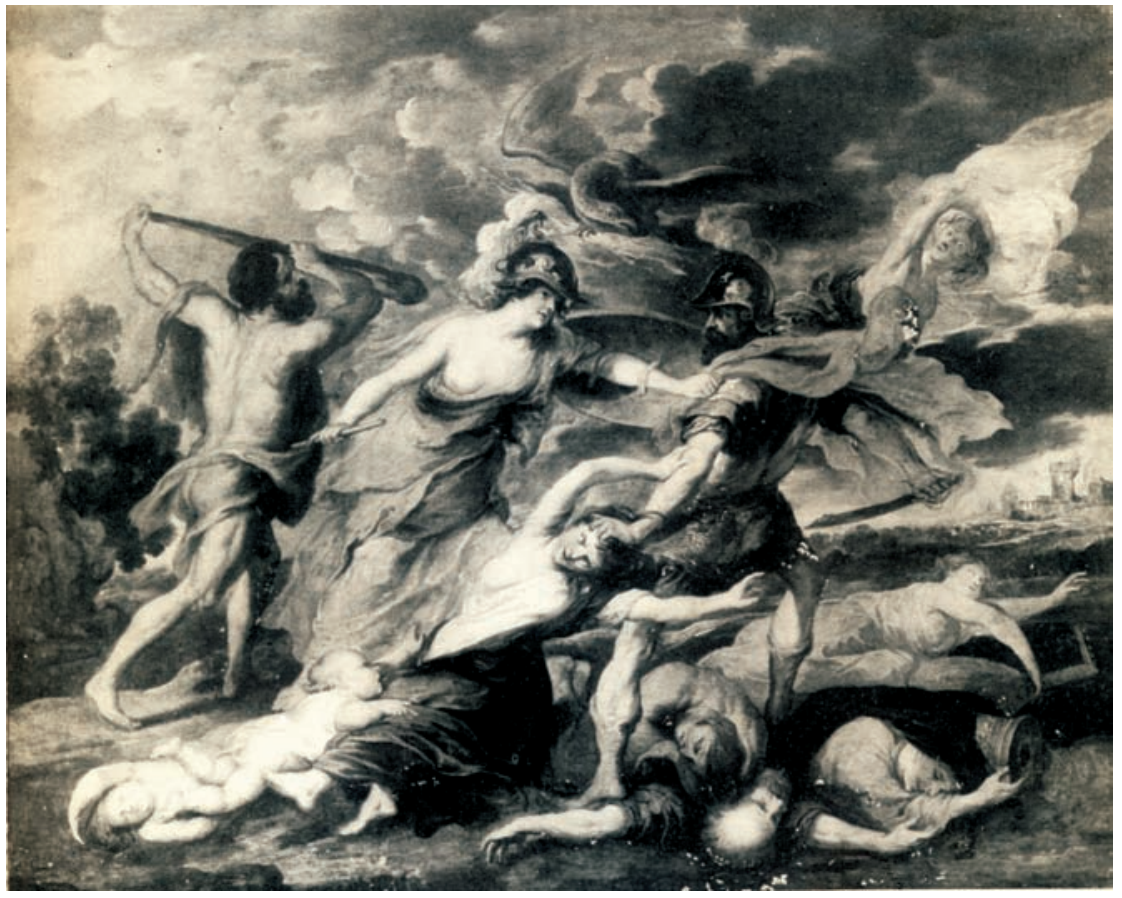

Fig. 3. Victor Wolfvoet, Hércules y Minerva enfrentándose a Marte, Sevilla.

Arch. esp. arte, LXXXV, 337, ENERO-MARZO 2012, 75-94, ISSN: 0004-0428 


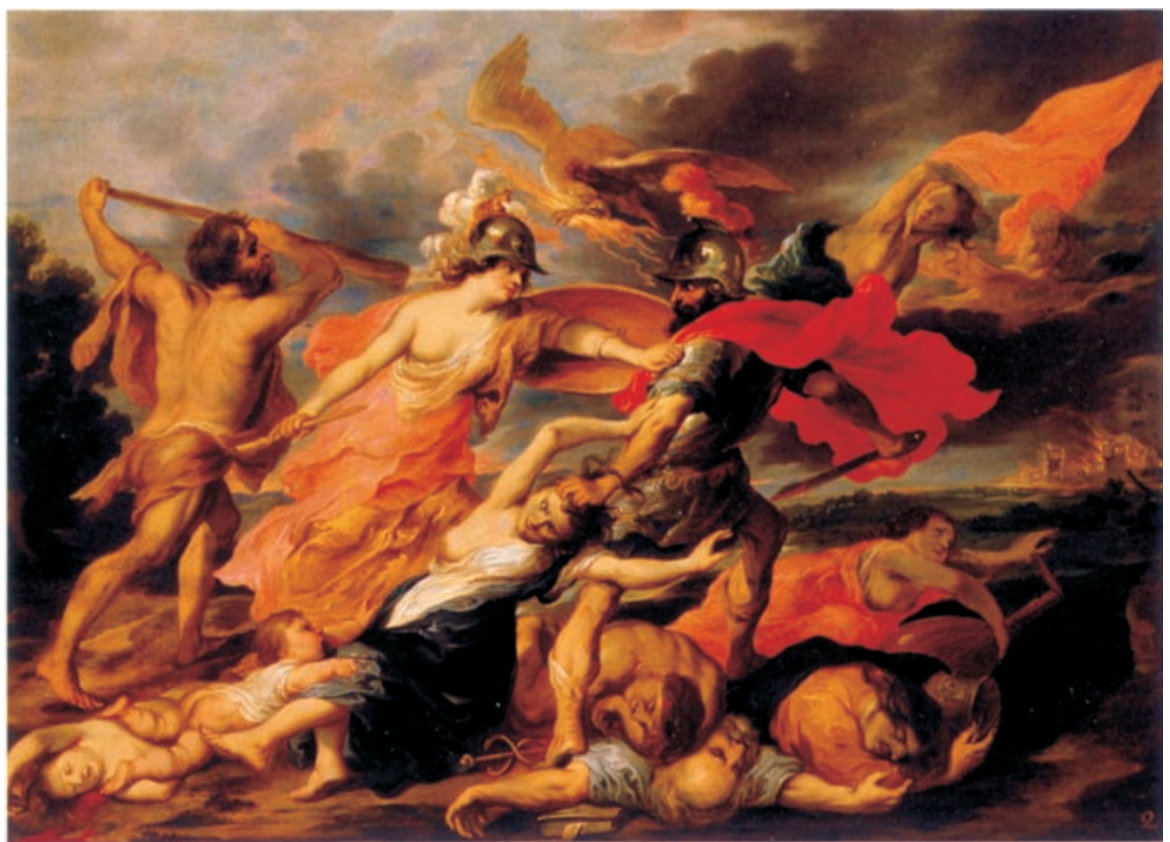

Fig. 4. Victor Wolfvoet, Hércules y Minerva enfrentándose a Marte, Museo Ermitage, San Petersburgo.

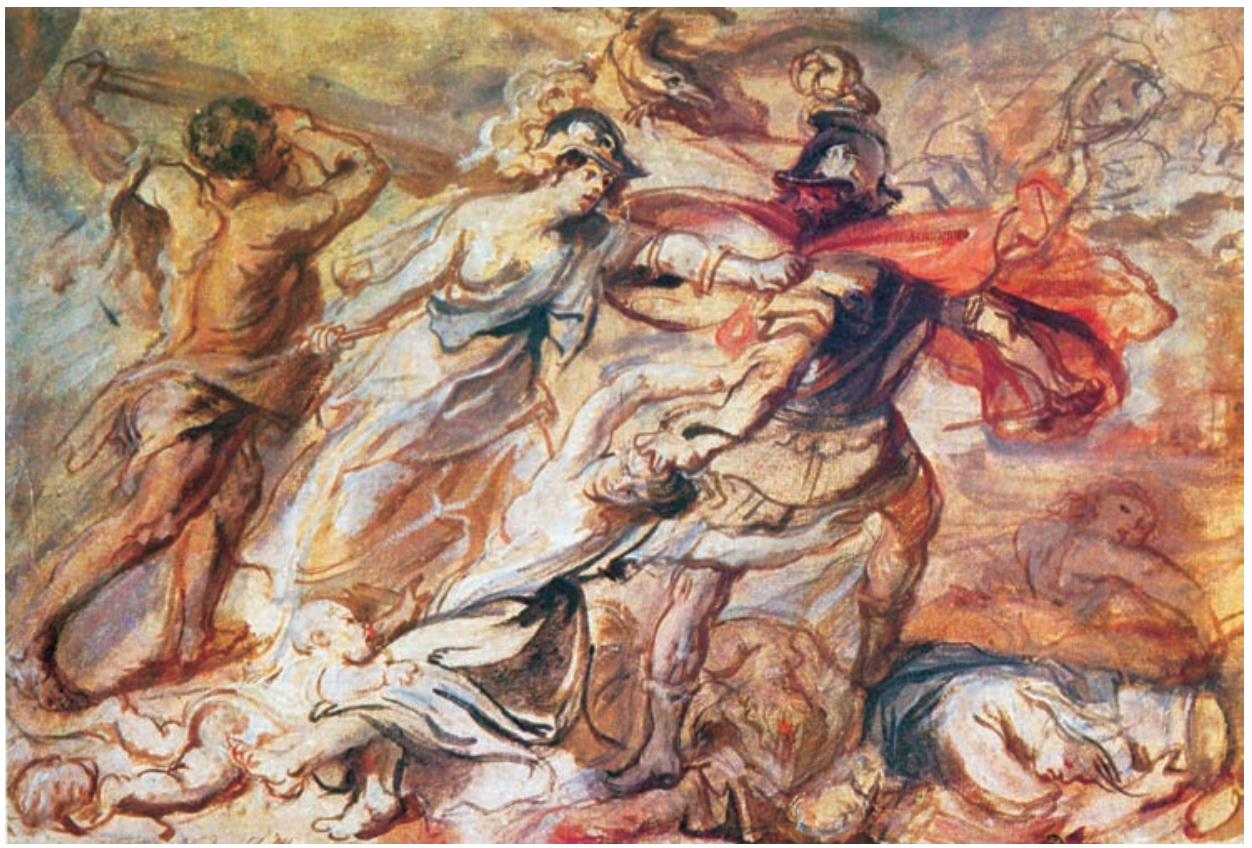

Fig. 5. Rubens, Hércules y Minerva enfrentándose a Marte, Museo del Louvre, París. 


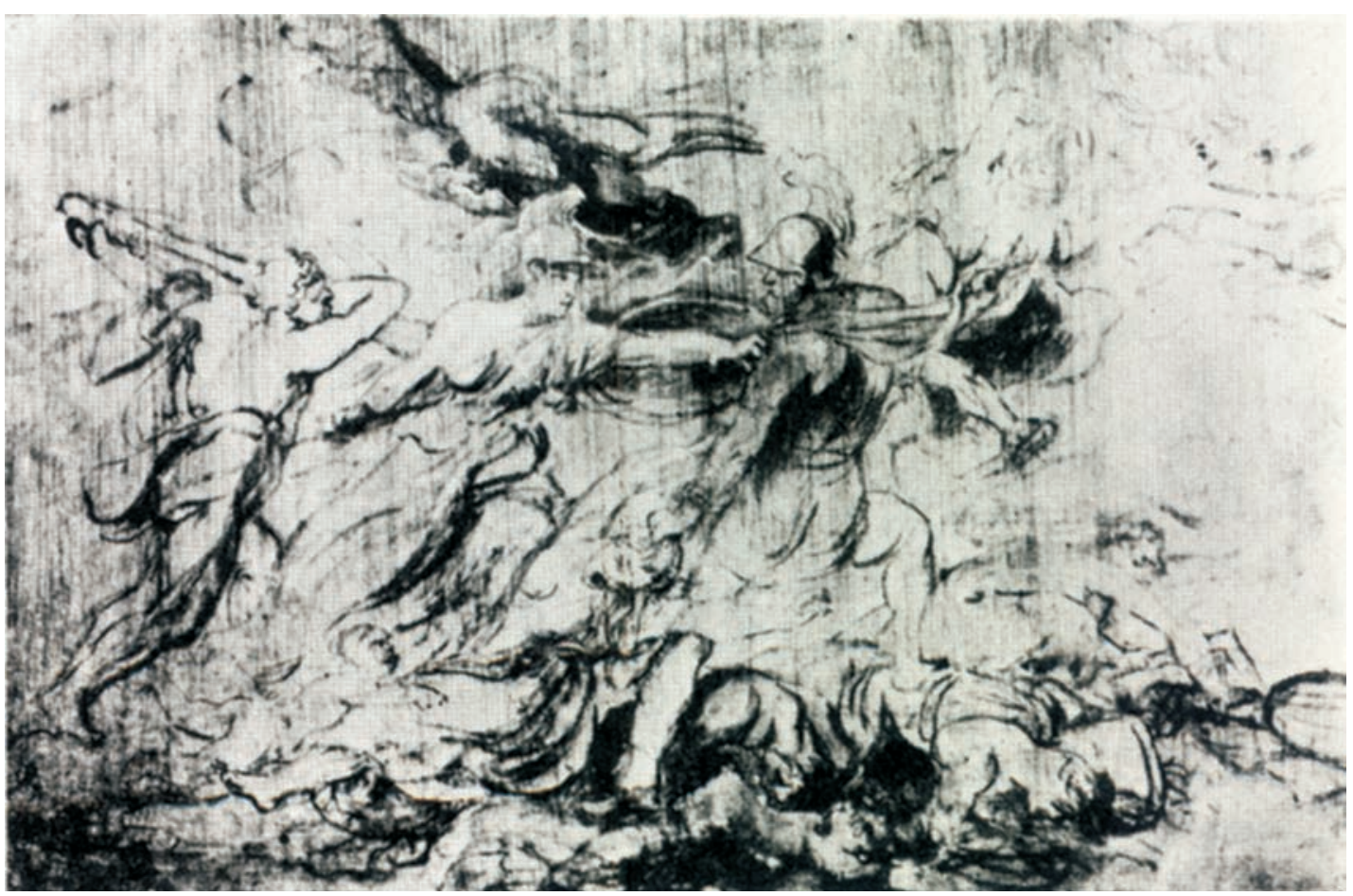

Fig. 6. Rubens, Hércules y Minerva enfrentándose a Marte, Museo de Amberes.

el Ermitage de San Petersburgo, también catalogado durante mucho tiempo entre los anónimos de la escuela de Rubens ${ }^{12}$ (fig. 4). Vemos como Victor Wolfvoet repite literalmente una composición de Rubens conocida a través de un dibujo conservado en el Museo del Louvre (fig. 5) ${ }^{13}$ y dos bocetos en los museos de Amberes (fig. 6) ${ }^{14}$ y Rotterdam ${ }^{15}$. Recientemente comentados por Anne-Marie $\operatorname{Logan}^{16}$, y muy útiles para el contenido iconográfico de las copias de Victor Wolfvoet. Tampoco estos bocetos de Rubens terminan en obras definitivas, según propuso el profesor Held. No hay variantes notorias entre el dibujo del Louvre y el boceto del museo de Amberes. Los dos distribuyen la historia en un formato apaisado, con los mismos personajes y el mismo espacio. Sólo hay diferencias de carácter más expresivo que formal, en el rostro de la mujer que arrastra a Marte por los cabellos al tiempo que los niños ruedan por tierra. Todo es idéntico al cobre que restituimos a Victor Wolfvoet, pero no tan literal a los bocetos de Amberes y Rotterdam citados. La cabeza de la mujer se vuelve gritando hacia el suelo con su boca abierta de dolor. El boceto de Rotterdam cambia al desplazar la composición vertical con Minerva girando su cuerpo para impedir la matanza del dios de la Guerra.

\footnotetext{
12 Gritsay y Babina, 2005: 160, n. ${ }^{\circ} 132$.

13 Rubens, Cat. Exp., París, 1978: 47, n. ${ }^{\circ} 28$.

14 Held, 1980: n. ${ }^{\circ} 244$.

15 Held, 1980: . $^{\circ} 253$

16 LOGAN, 2000: 176.
} 
Esto nos inclina a pensar que la fuente más directa de Victor Wolfvoet sería el dibujo del Gabinete de Estampas del Louvre. No es extraño, pues poseía varios bocetos y copias del maestro como consta detalladamente en su testamentaría ${ }^{17}$.

Añade más boscaje y muralla con torre a la izquierda, y las alas del águila de Júpiter que faltan en el dibujo. No descarto que esta última limitación se deba a la pérdida de algunos milímetros en los bordes del soporte, como revela la comparación con el boceto de Amberes. En fin, Victor Wolfvoet no logra el rictus de rabia contenida ni la energía de las manos de Minerva interponiéndose a Marte. El discípulo está lejos de la violencia incontenible del maestro. A la diosa protectora de las Ciencias y las Artes se unió el águila de Júpiter para ahuyentar la furia y el espanto con sus rayos; mientras agoniza la joven con el laúd en tierra. El laúd simboliza la música y la armonía; la escuadra y la columna rota, la medida y la arquitectura. La fatídica visión continúa más allá en las ruinas de una ciudad perdida en el horizonte.

\section{BIBLIOGRAFÍA}

Badouin, F., P. P. Rubens, 1977.

DíAz PADRÓN, La pintura flamenca del siglo XVII en España, Tesis doctoral, Ms. Universidad Complutense Madrid, 1976, XII tomos, T. IV.

DíAz PAdrón, M., "Dos cobres de Victor Wolfvoet en el Museo San Carlos de Méjico", Boletín Seminario de Estudios de Arte y Arqueología, Valladolid, n. ${ }^{\circ}$ 65. 1999, pp. 323-327.

DíAz PADRón, M., "Tres cobres restituidos a Victor Wolfvoet, el más fiel seguidor de Rubens", Archivo Español de Arte, t. 79, núm. 316, 2006, pp. 403-412.

Duverger, E., "Zeventiende-eeuwse schilderijen met de signatuur van Gilliam en Guillielmo Forchondt uit Antwerpen", Bull. Musées Royaux des Beaux-Arts de Belgique, núm. 1-3, 19891991, pp. 303-315.

Gritsay, N. y Babina, N., Gosudarstvenny Ermitazh. Flamandskaya Zhivopis. XVII-XVIII Verkov. Katalog kollertsii, St. Petersburg, 2005.

Hairs, M. L., Dans le Sillage de Rubens. Les Peintres d'Histoire aversois au XVII siècle, 1968.

Held, J. S., The Oil Sketches of Peter Paul Rubens, Princeton, 1980.

Hollstein, F. W. H. Hollstein's, Dutch and Flemish Etchings, Engravings and Woodcuts ca. 1450-1700, Rotterdam, 1995, II, XLVI.

Logan, A-M., Consistency and Change in Rubens's Drawings en Rubenianum concept, design \& execution in flemish painting (1550-1700), 2000.

Mesa J. De, y Gisbert, T., America. Bruid van de zon: 500 jaar Latijn-Amerika en de Lage Landen, cat. exp. Koninklij Museum voor Schone Kunsten, Amberes, 1992, pp. 182, 377.

Rooses, M. y Ruelens, R., Correspondance de Rubens et documents épistolaires concernant sa vie et ses oeuvres, VI, Amberes, 1887-1909.

Rubens, ses maîtres, ses élèves, dessins du mussée du Louvre. LXV Cat. Exp. Cabinet des Dessins. Paris, 1978.

Homenaje a Rubens en el IV Centenario de su nacimiento. Valdivieso, E. (comisario), cat. exp. Reales Alcázares. Sevilla, 1977-1978.

Wilmers, G., Cornelis Schut 1597-1655. A Flemish Painter of the High Baroque, Bélgica, 1996.

17 HAIRS, 1968: 221. 